\title{
Effect of counselling based on the PLISSIT model on pregnant women's sexual satisfaction: a randomised controlled trial
}

\author{
BEHNAZ NEJATI ${ }^{1, A-E}$, SEYEDEH ZAHRA MASOUMI ${ }^{2, A-G}$, PARISA PARSA $^{3, D}$, \\ ORCID ID: 0000-0002-2045-3707
}

MANOOCHEHR KARAMI ${ }^{4, \mathrm{c}}$, AZRA MORTAZAVI ${ }^{5, \mathrm{~F}}$

\author{
${ }^{1}$ Department of Midwifery, School of Nursing and Midwifery, Hamadan University of Medical Sciences, Hamadan, \\ Iran \\ ${ }^{2}$ Department of Midwifery, Mother and Child Care Research Center, School of Nursing and Midwifery, Hamadan \\ University of Medical Sciences, Hamadan, Iran \\ ${ }^{3}$ Chronic Disease Research Center, School of Nursing and Midwifery, Hamadan University of Medical Sciences, \\ Hamadan, Iran \\ ${ }^{4}$ Social Determinants of Health Research Center, Department of Epidemiology, School of Public Health, Hamadan \\ University of Medical Sciences, Hamadan, Iran \\ ${ }^{5}$ Emam Hossein Hospital, Hamadan University of Medical Sciences, Malayer, Iran
}

A - Study Design, B - Data Collection, C - Statistical Analysis, D - Data Interpretation, E - Manuscript Preparation, F - Literature Search, G - Funds Collection

Summary Background. Sexual satisfaction is often reduced during pregnancy owing to some sexual problems. Sexual counselling offered for pregnant women may reduce the complications of this disorder during pregnancy.

Objectives. This study was conducted to evaluate the effect of PLISSIT (permission, limited information, specific suggestions, and intensive therapy) model-based sexual counselling on pregnant women's sexual satisfaction.

Material and methods. In this randomised controlled clinical trial, the effect of PLISSIT model-based counselling on the sexual satisfaction of 80 pregnant women referred to health centres in the city of Malayer was investigated. The pregnant women were randomly assigned to intervention and control groups. The intervention group received counselling in 4 sessions of 45-90 minutes, and the control group received no counselling. Data collection tools were made up of demographic and Linda Berg questionnaires. Data analysis was conducted using SPSS-22 software, and a $p$-value lower than 0.05 was considered significant. The results were compared with repeated measurement, the independent $t$-test and chi-square test.

Results. Based on the results, the mean age of the mothers in the intervention and control groups was $26.32 \pm 3.92$ and $27.10 \pm 4.77$, respectively. There was a significant difference between the mean score of sexual satisfaction in both the intervention (64.50 \pm 7.19$)$ and control (58.90 \pm 11.92$)$ groups 2 weeks after the intervention, as well as 4 weeks after the intervention in both the intervention $(69.65 \pm 5.51)$ and control $(60.05 \pm 13.96)$ groups, respectively.

Conclusions. Sexual satisfaction in pregnancy can be enhanced by providing PLISSIT-based sexual counselling. Key words: orgasm, pregnant womec, PLISSIT model.

Nejati B, Masoumi SZ, Parsa P, Karami M, Mortazavi A. Effect of counselling based on the PLISSIT model on pregnant women's sexual satisfaction: a randomised controlled trial. Fam Med Prim Care Rev 2020; 22(1): 43-48, doi: https://doi.org/10.5114/fmpcr. 2019.90163.

\section{Background}

Sexual satisfaction is one of the indicators of mental health and life expectancy, which is regarded as objective sentiment, pleasure, acquiescence and enjoyment by men and women [1]. Painless and satisfactory sexual activity (which is a crucial component of establishing relationships in many couples) is often influenced by the physical and psychological needs of pregnancy [2]. The effects of these factors on the sexual relationship of a pregnant woman can be very different and reflect the unique nature of human sexuality [3]. Women undergo various unwanted changes during pregnancy, including changes in their physical and mental needs. The physical, emotional and economic stress of pregnancy may negatively affect emotional and sexual intimacy. Attitudes and sexual behaviours during pregnancy are influenced by sexual value systems, native culture, traditional beliefs, religious beliefs, physical changes and medical constraints [4]. During pregnancy, sexual satisfaction may remain unchanged, or it may increase or decrease. Each of these changes can lead to inefficient long-term sexual and mental health in mothers [5]. Most of the sexual problems in pregnancy are due to misconceptions and misunderstandings about the physical and emotional changes during this period [6]. Sexual satisfaction during pregnancy and sexual information about this period are significantly associated with each other in many aspects, and most people do not receive sufficient information from health providers during pregnancy $[7,8]$. Paying attention to the sexual health of couples, recognising their concerns, helping to address these concerns and improving sexual function should be the mainstream of care during pregnancy [9]. In addition, individuals must learn the skills needed for satisfactory sexual relationships. This can be achieved through counselling and training, which can lead to increased sexual satisfaction [10]. Providing accurate and scientific information on physical and mental changes during pregnancy through health personnel, especially midwives, as well as providing sexual counselling in pae- 
diatric education, are extremely helpful [11]. One of the ways to improve sexual satisfaction is counseling and training based on the PLISSIT model. This model is the most commonly used tool to evaluate performance and ultimate sexual satisfaction, which can be used by all people [12]. This model, consisting of 4 levels of interventional therapy, is a useful tool for counselling and sex intervention, and using the first 3 levels of this model, approximately $80-90 \%$ of sexual concerns can be addressed [4]. Studies have shown that using this model reduces sexual problems in women [13]. Pregnancy is a critical period in women's lives. This experience has important and long-term effects on women and their families $[14,15]$. Sexual problems, such as lack of orgasm, vaginal dryness, sexual desire and coldness, and some emotional factors like fear of foetal injury and acceptance of a new role, affect sexuality during pregnancy. Dysfunction and sexual dissatisfaction are one of the most common problems during pregnancy, affecting a large number of pregnant women. Midwifery counselling helps couples to have better sexual relationships by providing information, as well as understanding the problem, examining and analysing it and helping them to decide and choose the right solution.

Given that limited studies have been conducted to support the effect of sexual counselling on the sexual satisfaction and marital satisfaction of pregnant women, the present study was conducted to determine the effect of PLISSIT model-based counselling on pregnant women's sexual satisfaction.

\section{Objectives}

This study was conducted to evaluate the effect of PLISSIT model-based sexual counselling on pregnant women's sexual satisfaction.

\section{Material and methods}

\section{Study design, setting}

This research was a randomised controlled trial study conducted in 2015 in the city of Malayer, Hamadan province, Iran.

\section{Sampling method}

In this study, 80 people were examined (40 in the intervention group and 40 in the control group). The sample size was based on the data provided by Rostamkhani et al. [16]. The final sample size was determined to be 40 in each group (intervention and control).

Considering that there are 5 urban centres in Malayer, out of these centres, 2 centres were randomly allocated to the control group, and 2 centres were assigned to the intervention group. The selection of individuals in the centres was based on random numbers (Figure 1).

In each clinic, eligible people were given a number and placed via the lottery method in 2 groups.

\section{Participants}

The study inclusion criteria included a gestational age of 24 to 26 weeks, an age range of $18-35$ years, having purely female sexual dysfunction with confirmation of a psychiatrist and no history of sexual dysfunction before pregnancy. The study exclusion criteria included the occurrence of any pregnancy complications during the study, such as abortion, placental abruption, abnormal foetal position, bleeding, diabetes, hypertension, preterm labour, abnormal foetal heart patterns and decreased foetal movements.

\section{Study instruments}

In this study, sexual satisfaction was measured using Linda Berg's sexual satisfaction questionnaire. The questionnaire was prepared by Linda Berg and Cresy in 1997. Likert scaling is as follows: always $=5$, most of the times $=4$, sometimes $=3$, rarely $=2$, never $=1$. The maximum score is 85 , and the lowest score is 17 . The validity of this questionnaire was confirmed by Salehy Fadardy in 1999 [17]. The reliability of this questionnaire was confirmed by Cronbach Alpha 0.94 .

All of the pregnant women meeting the criteria for entering the study gave written consent for participation. The participants of both the intervention and control groups completed the Linda Berg sexual satisfaction questionnaire before the intervention.

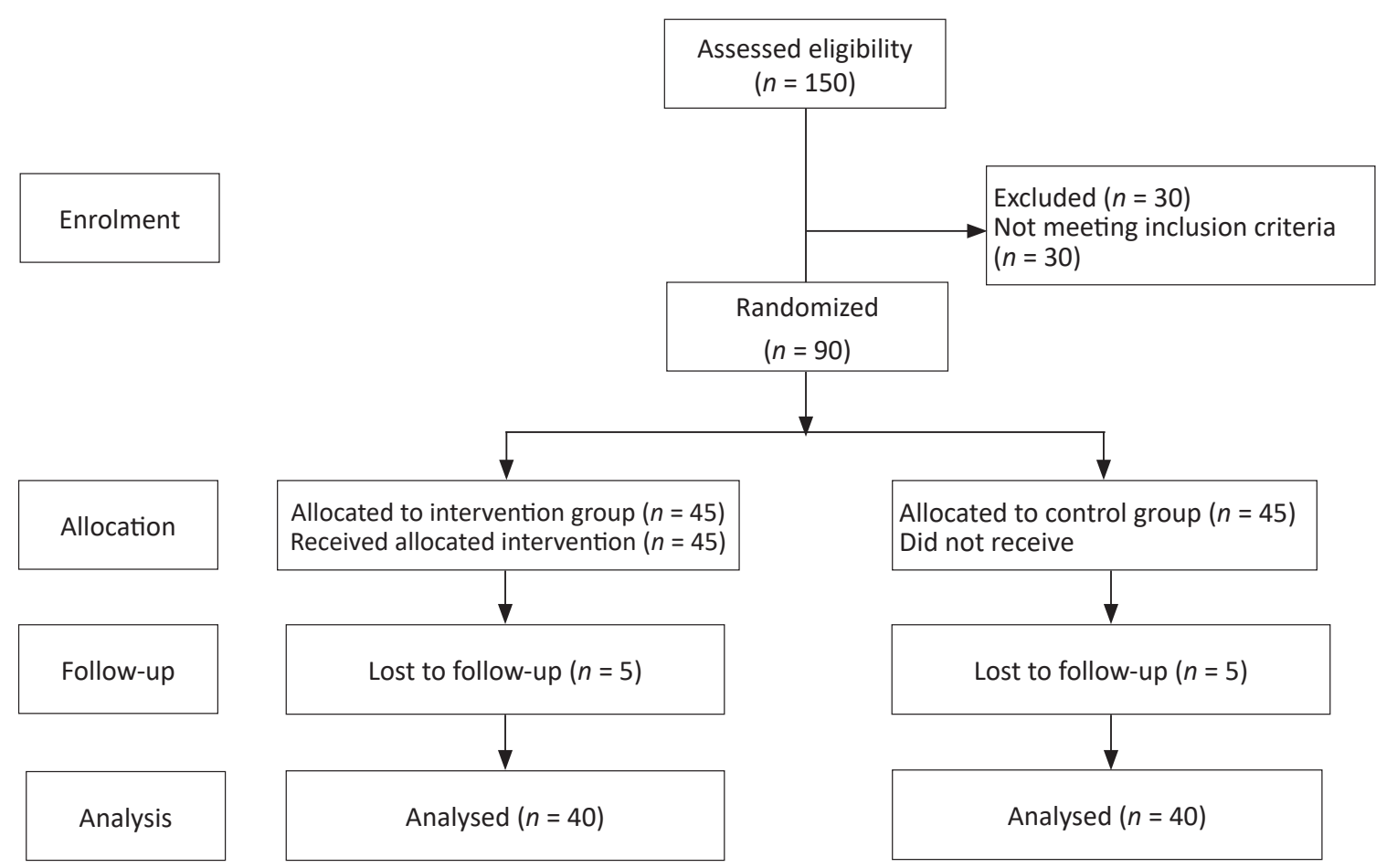

Figure 1. The process of selecting samples 


\section{Intervention}

The control group received routine consultation from the midwife of the clinic within a month, while the intervention group, in addition to routine care, received sexual counselling based on the PLISSIT model. This model has 4 steps, as follows:

First level (permission): The patient is allowed to share information about her sex activity. Thus, any concerns are identified [4]. Second level (limited information): Real information is provided in response to a patient's question or experience. Health service providers attempt to correct the mistakes and lack of information in this regard. This is used to solve non-complex problems [18]. Third level (specific suggestions): This stage of counselling involves offering specific suggestions to a pregnant woman and requires a higher level of expertise. Sexual satisfaction is strongly linked to the sexual relationship between a man/ /woman with a sexual disorder and his/her spouse. At this stage, it is highly important to evaluate couples' sexual relations and provide multiple methods to express sexual needs [19]. Fourth level (referral for specialised care): This intervention should be conducted in the case of severe or prolonged sexual problems. It is necessary to train healthcare providers and, if necessary, referral patients for appropriate care to healthcare professionals [12].

The counselling session was conducted weekly in 4 sessions of $45-90$ minutes by a trained midwife. The content of the training sessions at the first session included: introducing the counsellor and participants to each other, explaining the details of counselling sessions and their goals and familiarising the participants with the PLISSIT model, as well as anatomy and maternal physiology during pregnancy (Level 1 of the PLISSIT model). The participants were asked to address their sexual concerns in this regard, and the counsellor responded to questions from clients based on the PLISSIT model and described the various sex positions during pregnancy. The limitations of sex during this period and the health issues needed during sex in pregnancy were explained. Specific suggestions were offered (Levels 2 and 3 of the PLISSIT model). The following-up of the PLISSIT counselling steps for the second session was to examine the areas of sexual function and its changes during pregnancy (Level 1 of the PLISSIT model). The midwife answered the participants' questions, and the 4 phases of the sexual response cycle, as well as the physical and mental differences between men and women, were expressed at each stage of the sexual cycle. Incorrect beliefs about sex during pregnancy were corrected (Levels 2 and 3 of the PLISSIT model). Finally, the consultation session was finalised and planned for the next meeting. In the third session, the pattern of sexual changes during pregnancy was explained, and the counsellor responded to the questions asked by the patients. The protocol for sexual activity changes during pregnancy was reviewed (Levels 2 and 3 of the PLISSIT model), and finally, a counselling session was scheduled, and a follow-up meeting was planned. In the fourth session, sexual satisfaction and subsequent marital satisfaction in pregnancy were explained. The counsellor's response to the questions about the effective factors of sexual satisfaction and marital satisfaction in pregnancy was then answered, and the counselling process was finalised (Levels 2 and 3 of the PLISSIT model). It should be noted that the content of the training sessions was presented to the recipients in a pamphlet. The researcher also had a weekly phone call to assess the sexual satisfaction of the intervention group. For the post-test, both groups (intervention and control) completed the questionnaire 2 and 4 weeks after completion of the consultation. The psychiatrist examined the pregnant women of both groups before the study, 2 and 4 weeks after the intervention.

\section{Ethical considerations}

The study protocol was approved by the Ethics Committee of the Hamadan University of Medical Sciences (IR.UMSHA.REC. 9407284056). The protocol was also registered in the Iranian Registry for Randomised Controlled Trials (IRCT2015070713405N11) and was conducted after obtaining the permission of the Deputy of the Hamadan University of Medical Sciences. This article is the result of a master's thesis on midwifery counselling.

\section{Statistical analysis}

The independent $t$-test was used to comparing means between the intervention and control groups. Chi-square and $t$-tests were used to assess demographic variables in both groups and the one-way repeated-measures ANOVA test was used to examine the effects of intervention at different times. $p$-values less than 0.05 were considered statistically significant. Data was analysed using the SPSS-22 software.

\section{Results}

\section{Findings related to socio-demographic characteristics}

In this study, 80 pregnant women having the inclusion criteria (40 patients in each group) were evaluated. Investigation

\begin{tabular}{|c|c|c|c|c|}
\hline \multicolumn{2}{|l|}{ Variables } & Intervention group & Control group & $p$ \\
\hline \multicolumn{2}{|c|}{ Age (years), Mean (Standard Deviation) } & $26.32(3.92)$ & $27.10(4.77)$ & 0.43 \\
\hline \multicolumn{2}{|c|}{ Gestational age, Mean (Standard deviation) } & $24.77(0.83)$ & $24.77(0.80)$ & 1.00 \\
\hline \multicolumn{2}{|c|}{ Gravity, Mean (Standard Deviation) } & $1.62(0.70)$ & $1.97(1.04)$ & 0.08 \\
\hline \multirow[t]{2}{*}{ Employment status $n(\%)$} & employed & $4(10)$ & $6(15)$ & \multirow[t]{2}{*}{0.73} \\
\hline & housewife & $36(90)$ & $34(85)$ & \\
\hline \multirow{4}{*}{$\begin{array}{l}\text { Education } \\
n(\%)\end{array}$} & elementary & $3(7.5)$ & $3(7.5)$ & \multirow[t]{4}{*}{0.89} \\
\hline & guidance school & $3(7.5)$ & $5(12.5)$ & \\
\hline & high school & $17(42.5)$ & $17(42.5)$ & \\
\hline & academic and higher education & $17(42.5)$ & $15(37.5)$ & \\
\hline \multirow{4}{*}{$\begin{array}{l}\text { Husband's education } \\
n(\%)\end{array}$} & elementary & $3(7.5)$ & $4(10)$ & \multirow[t]{4}{*}{0.59} \\
\hline & guidance school & $4(10)$ & $8(20)$ & \\
\hline & high school & $14(35)$ & $16(40)$ & \\
\hline & academic and higher education & $14(35)$ & $17(42)$ & \\
\hline \multirow{2}{*}{$\begin{array}{l}\text { Husband's employment status } \\
\text { n (\%) }\end{array}$} & employed & 39 (97.5) & 39 (97.5) & \multirow[t]{2}{*}{1.00} \\
\hline & unemployed & $1(2.5)$ & $1(2.5)$ & \\
\hline \multirow{2}{*}{$\begin{array}{l}\text { Desire for this pregnancy } \\
n(\%)\end{array}$} & yes & $27(67.5)$ & 31 (77.5) & \multirow[t]{2}{*}{1.00} \\
\hline & no & $13(32.5)$ & $9(22.5)$ & \\
\hline
\end{tabular}


of quantitative variables using the Kolmogorov-Smirnov test showed that all variables had normal distribution. The mean age of the intervention and control groups was $26.32 \pm 3.92$ and $27.10 \pm 4.77$ years, respectively, and this difference was not statistically significant. The comparison of the 2 groups did not show any significant difference in terms of other variables; therefore, pregnant women were matched in both the intervention and control groups (Table 1).

\section{Intervention effect}

The comparison of means of sexual satisfaction before, 2 weeks after and 4 weeks after the intervention in the control and intervention groups showed a significant difference between the 2 groups $(p<0.001)$. In addition, the comparison of the 2 groups in 3 stages showed that there was no significant difference between the 2 groups in terms of sexual satisfaction score in the pre-test ( $p=0.59$ ), but there was a significant difference between the 2 groups after 2 and 4 weeks of intervention ( $p=0.01$ and $p<0.001$, respectively) (Table 2 ).

\begin{tabular}{|c|c|c|c|c|}
\hline $\begin{array}{l}\text { Sexual sat- } \\
\text { isfaction } \\
\text { Score } \\
\text { of the } 2 \\
\text { groups }\end{array}$ & $\begin{array}{l}\text { Before the } \\
\text { interven- } \\
\text { tion } \\
\text { M (SD) }\end{array}$ & $\begin{array}{l}2 \text { weeks } \\
\text { after the } \\
\text { interven- } \\
\text { tion } \\
\text { M (SD) }\end{array}$ & $\begin{array}{l}4 \text { weeks } \\
\text { after the } \\
\text { interven- } \\
\text { tion } \\
\text { M (SD) } \\
\end{array}$ & $\begin{array}{l}\text { One-way } \\
\text { repeated } \\
\text { measures } \\
\text { ANOVA }\end{array}$ \\
\hline $\begin{array}{l}\text { Interven- } \\
\text { tion }\end{array}$ & $\begin{array}{l}56.82 \\
(9.83)\end{array}$ & $\begin{array}{l}64.50 \\
(7.19)\end{array}$ & $\begin{array}{l}69.65 \\
(5.51)\end{array}$ & \multirow[t]{2}{*}{$\begin{array}{l}p>0.001 \\
\mathrm{~F}=61.22\end{array}$} \\
\hline Control & $\begin{array}{l}58.25 \\
(13.38) \\
\end{array}$ & $\begin{array}{l}58.90 \\
(11.92) \\
\end{array}$ & $\begin{array}{l}60.05 \\
(13.96) \\
\end{array}$ & \\
\hline$p^{* *}$ & $\begin{array}{l}0.59 \\
T=0.54\end{array}$ & $\begin{array}{l}0.01 \\
T=2.54\end{array}$ & $\begin{array}{l}p>0.001 \\
T=4.40\end{array}$ & \\
\hline
\end{tabular}

** $\mathrm{T}-$ independent.

\section{Discussion}

Based on the present study results, sexual counselling during pregnancy could be effective in increasing pregnant women's sexual satisfaction. The results of the present study were consistent with those of Schulz's study. Schulz's study was a randomised clinical trial study examining 30-year-old women from the third trimester of pregnancy to 66 months postpartum. The study results indicated that preventive intervention could maintain marital quality when becoming a parent. Couples who did not receive intervention experienced reduced sexual and marital satisfaction [20]. The results of Banaei's et al. study, which were in line with the increasing impact of counselling on sexual satisfaction, are consistent with the present study results [21]. The present study indicated that counselling could be useful in increasing sexual satisfaction during pregnancy. Furthermore, in the study conducted by Pakgohar et al., sexual counselling for infertile women in 2 stages before and 3 months after intervention showed a significant difference between the mean sexual and marital satisfaction of couples [22].

Masoumi et al., in their study, concluded that sexual education or marital counselling promoted family health, reduced sexual violence in the family, prevented sexually transmitted diseases and provided a positive attitude toward sexual relations, sexual pleasure, reduced family inequity and sexual satisfaction. As a result, marital satisfaction is attained by couples [23]. Alidost et al., in their study, demonstrated that after sexual counselling, the mean score of sexual satisfaction in the posttest and the 6-month follow-up was significantly higher than that of the pre-test score [9]. The present study results are similar to the above findings and suggest that PLISSIT model-based sexual counselling increases sexual satisfaction.
The present study showed that lack of sexual counselling and intervention in the control group did not show any changes in sexual satisfaction, and thus the results showed no significant difference in each stage compared to the previous one. Our research was in line with the study by Babazadeh et al., in which there was a significant difference between the sexual satisfaction of women in the intervention group before and 2 months after education, while in the control group, there was no significant difference [24].

The results of this study have been consistent with those of the study by Parva et al. on comparing the educational programme on sexual satisfaction in intervention and control groups. The intervention group's couples participated in sessions for 4 consecutive weeks, 2 sessions a week, and a control group was also considered. The groups were evaluated before and after the training sessions. The results showed that spouses who participated in the sexual enhancement programme had more pleasure in their sexual relationship than those in the other group. In addition, the participants in the sexual enhancement programme felt a greater amount of affection, and their overall marital satisfaction improved [25]. Rosier and Tyler showed that sexual skills training could lead to positive emotions, better proximity and sincerity of the spouses, as well as promotion of marital relationships. If continued, it increases the ability to solve conflicts between couples and increases marital satisfaction [26]. Byers investigated the causal link between sexual communication and marital satisfaction and concluded that there was a two-way correlation between sexual satisfaction and marital satisfaction. Their study also showed that sexual satisfaction resulting from counselling based on the PLISSIT model could significantly affect pregnant women's sexual satisfaction, being consistent with the results of our study [27]. The present study is also consistent with the study of Sabeti et al., who investigated the effect of educational intervention on women's sexual function. A total of 130 women participated in 2 intervention and control groups (each one 65 individuals). The intervention group was provided with 2 training sessions of 180 minutes, and the results showed that sexual health training could improve sexual function in women $[28,29]$. Abdelhakm et al. concluded that sexual performance counselling in diabetic women using the 4 levels of the PLISSIT model and the FSFI questionnaire was effective in promoting female sexual function [30]. Banaei et al., in their study, indicated that PLISSIT modelbased sexual performance counselling in breastfeeding women, even after 3 months of intervention, was effective in increasing female sexual function [31]. Chun, using the PLISSIT model to increase the sexual function of women with gynaecological cancer, concluded that there was a significant statistical difference in FSFI sub-domains, including sexual desire, arousal, lubrication, orgasm and satisfaction, but not pain, and women's sexual function will improve with this model [32]. Overall, the results of these studies were consistent with those of our study.

\section{Limitations of the study}

In this study, the duration of the follow-up was short, which can be considered a limitation.

\section{Conclusions}

It can be concluded that PLISSIT model-based sexual counselling significantly affects sexual satisfaction improvement. This method prevents various sexual disorders during this period.

Acknowledgments. This paper was one part of an MSc thesis and a research project under number: IR, UMSHA, REC,1394.225. Financial support was provided by the deputy vice-chancellor for research affairs of the Hamadan University of Medical Sciences. 
Source of funding: This paper was developed using funds provided by the Hamadan University of Medical Sciences. Conflicts of interest: The authors declare no conflicts of interest.

\section{References}

1. Najafizadeh A, Samaeelvand N, Yousefi H, et al. A comparison between the attachment styles and Mate-selection criteria in Urban and rural girls. ILSHS 2015; 47: 1-9, doi: 10.18052/www.scipress.com/ILSHS.47.1.

2. Efe H, Bozkurt M, Sahin L, et al. The effects of pregnancy on the sexual life of Turkish women. Proc Obstet Gynecol 2014; 4(1): 1-11.

3. Sossah L. Sexual behavior during pregnancy: a descriptive correlational study among pregnant women. European Journal of Research in Medical Sciences 2014; 2(1): 16-27.

4. Berek JS. Berek and Novak's Gynecology. 16 th ed. Philadelphia: Lippincott Williams and Wilkins; 2019.

5. Latifnejad Roudsari R, Moradi M, Esmaily H. Effect of sexual counseling on stress, anxiety, and depression in women during postpartum period. Evidence Based Care 2017; 7(2): 17-26.

6. Khalesi ZB, Bokaie M, Attari SM. Effect of pregnancy on sexual function of couples. Afr Health Sci 2018; 18(2): 227-234.

7. Abouzari-Gazafroodi K, Najafi F, Kazemnejad E, et al. Demographic and obstetric factors affecting women's sexual functioning during pregnancy. Reprod Health 2015; 12(1): 72, doi: 10.1186/s12978-015-0065-0.

8. Masoumi SZ, Garousian M, Khani S, et al. Comparison of quality of life, sexual satisfaction and marital satisfaction between fertile and infertile couples. Int J Fertil Steril 2016; 10(3): 290-296.

9. Alidost F, Dolatian M, Shams J, et al. The correlation of sexual dysfunction with prenatal stress and quality of life: a path analysis. Iran Red Crescent Med J 2017; 19(7): e55686, doi: 10.5812/ircmj.55686.

10. Schofield MJ, Mumford N, Jurkovic D, et al. Short and long-term effectiveness of couple counselling: a study protocol. BMC Public Health 2012; 12(1): 735, doi: 10.1186/1471-2458-12-735.

11. Heidari $\mathrm{M}$, Aminshokravi $F$, Zayeri $F$, et al. Effect of sexual education on sexual function of iranian couples during pregnancy: a quasi experimental study. J Reprod Infertil 2018; 19(1): 39-48.

12. Allen L, Fountain L. Addressing sexuality and pregnancy in childbirth education classes. J Perinat Educ 2007; 16(1): 32-36.

13. Farnam F, Janghorbani M, Raisi F, et al. Compare the effectiveness of PLISSIT and sexual health models on Women's sexual problems in Tehran, Iran: a randomized controlled trial. J Sex Med 2014; 11(11): 2679-2689.

14. Oshvandi K, Keshmiri K, Salavati M, et al. Effectiveness of education based on Orem's self-care model in self-care activity of patients with implantable cardioverter defibrillators. Hayat 2014; 19(3): 47-55.

15. Khodaveisi M, Faal Araghi Nejad A, et al. Comparing the structural standards of nursing homes in Markazi Province, Iran with international standards. Iranian Journal of Ageing 2018; 13(3): 362-371.

16. Rostamkhani F, Ozgoli G, Khoei EM, et al. Effectiveness of the PLISSIT-based Counseling on sexual function of women. Adv Nurs Midwifery 2012; 22(76): 1-9.

17. Salehy Fadardy J. The development and validation of marital satisfaction questionnaire on a sample of students of Ferdowsi University. Psychother Novelties 1999; 4(13): 84-93.

18. Nobre PJ, Pinto-Gouveia J, Gomes FA. Prevalence and comorbidity of sexual dysfunctions in a Portuguese clinical sample. J Sex Marital Ther 2006; 32(2): 173-182.

19. Zarbo C, Tasca GA, Cattafi F, et al. Integrative psychotherapy works. Front Psychol 2016; 6: 2021, doi: 10.3389/fpsyg.2015.02021.

20. Schulz MS, Cowan CP, Cowan PA. Promoting healthy beginnings: randomized controlled trial of a preventive intervention to preserve marital quality during the transition to parenthood. J Consult Clin Psychol 2006; 74(1): 20-31.

21. Banaei M, Safarzadeh S, Shahrahmani H. Impact of counseling on sexual intimacy of lactating women. Ann Trop Med Public Health 2017; 10: 1763-1769.

22. Pakgohar $\mathrm{M}$, Vizheh $\mathrm{M}$, Babaee $\mathrm{G}$, et al. Effect of counseling on sexual satisfaction among infertile women referred to Tehran fertility center. Hayat 2008; 14(1): 21-30.

23. Masoumi SZ, Kazemi F, Nejati B, et al. Effect of sexual counseling on marital satisfaction of pregnant women referring to health centers in Malayer (Iran): an educational randomized experimental study. Electron Physician 2017; 9(1): 3598-3604.

24. Babazadeh R, Mirzaei Najmabadi K, Masoumi Z, et al. Investigating effect of teaching on sexual-activity changes during pregnancy. Iranian J Obstetrics, Gynecology and Infertility 2012; 15(12): 18-26.

25. Parva M, Lotfi R, Nazari MA, et al. The effectiveness of sexual enrichment counseling on sexual assertiveness in married women: a randomized controlled trial. Shiraz E Medical Journal 2018; 19(1): e14552.

26. Rosier JG, Tyler JM. Finding the love guru in you: examining the effectiveness of a sexual communication training program for married couples. Marriage \& Family Review 2017; 53(1): 65-87.

27. Byers ES. Relationship satisfaction and sexual satisfaction: a longitudinal study of individuals in long-term relationships. J Sex Res 2005; 42(2): 113-118.

28. Sabeti F, Sadat-Tavafian S, Zarei F. The effect of educational intervention on sexual function of women referred to Health center of southern Tehran. Nurs Pract Today 2018; 5(2): 280-289.

29. Khalili A, Shayan A, Khodaveisi M, et al. Construction of professional ethics questionnaire in midwifery. IJFMT 2017; 11(2): 237-240.

30. Abdelhakm EM, Said AR, Elsayed DMS. Effect of PLISSIT model sexual counseling program on sexual quality of life for postpartum women. Am J Nurs Sci 2018; 7(2): 63-72.

31. Banaei M, Tork Zahrani S, Pormehr-Yabandeh A, et al. Investigating the impact of counseling based on PLISSIT model on sexual intimacy and satisfaction of breastfeeding women. Int J Pharm Res Allied Sci 2016; 5(3): 489-499.

32. Chun N. Effectiveness of PLISSIT model sexual program on female sexual function for women with gynecologic cancer. J Korean Acad Nurs 2011; 41(4): 471-80.

Tables: 2

Figures: 1

References: 32 
Address for correspondence:

Seyedeh Zahra Masoumi, PhD, Assoc. Prof.

Department of Midwifery

Mother and Child Care Research Center

School of Nursing and Midwifery

Hamadan University of Medical Sciences

Hamadan, Iran

Tel.: +98 8138380150

E-mail: zahramid2001@yahoo.com 